I. Higuchi and M. Itô

Nagoya Math. J.

Vol. 53 (1974), 127-135

\title{
ON THE THEOREM OF KISHI FOR A CONTINUOUS FUNCTION-KERNEL
}

\author{
ISAO HIGUCHI AND MASAYUKI ITÔ
}

\section{Introduction}

In the potential theory with respect to a non-symmetric functionkernel, the following theorem is obtained by M. Kishi ([3]).

Let $X$ be a locally compact Hausdorff space and $G$ be a lower semicontinuous function-kernel on $X$. Assume that $G(x, x)>0$ for any $x$ in $X$ and that $G$ and the adjoint kernel $\breve{G}$ of $G$ satisfy "the continuity principle". Then the following four statements are equivalent.

(1) $G$ satisfies the domination principle.

(2) $G$ satisfies the domination principle.

(3) $G$ satisfies the balayage principle.

(4) $G$ satisfies the balayage principle.

In the class of lower semi-continuous function-kernels on $X$, the subclass of continuous function-kernels on $X$ is essential for the continuity principle. We remark that the continuity principle follows from a certain maximum principle.

A function $G$ defined in the product space $X \times X$ is called a continuous function-kernel if $G$ is non-negative, continuous in the extended sense and finite outside the diagonal set of $X \times X$.

In this paper, we shall prove the continuity principle for $\mathscr{G}$ follows from the domination principle for $G$ under a certain additional condition. On the other hand, it is well-known that the domination principle for $G$ implies the continuity principle for $G$ itself. Using our result, we shall obtain that the above theorem is valid in the case of a continuous function-kernel on $X$ without the assumption that $G$ and $\breve{G}$ satisfy the continuity principle. In the proof, we shall use a result of one of the authors (cf. [2]), and the following proposition will be essential in our proof.

Received August 8, 1973. 
Let $G$ be a continuous function-kernel on $X$ satisfying the domination principle. Assume that every point of $X$ is non-isolated and that $G(x, x)>0$ for any $x$ in $X$ and for any non-empty open set $\omega$ in $X$, the $G$-capacity of $\omega$ is positive. Then $G(x, y)>0$ in $X \times X$.

We remark that $M$. Kishi first proved the above equivalence for a strictly positive function-kernel (cf. [3]).

\section{Preliminaries}

Let $X$ be a locally compact Hausdorff space and $\Delta$ denote the diagonal set of the product space $X \times X$. A function $G$ defined everywhere in $X \times X$ is called a function-kernel if $G$ is non-negative and Borel measurable ([2]). The function-kernel $\check{G}$ on $X$, defined by $\check{G}(x, y)=$ $G(y, x)$ for any $(x, y)$ in $X \times X$, is called the adjoint kernel of $G$. For a positive Radon measure $\mu$ in $X$, the potential $G \mu$ and the adjoint potential $\breve{G}_{\mu}$ of $\mu$ are defined by

$$
G \mu(x)=\int G(x, y) d \mu(y) \text { and } \check{G} \mu(x)=\int \check{G}(x, y) d \mu(y) .
$$

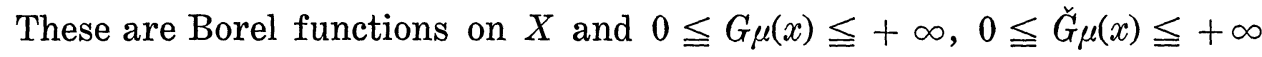
in $X$. The $G$-energy of $\mu$ is defined by $\int G \mu d \mu$. Evidently the $G$-energy of $\mu$ is equal to the $\check{G}$-energy of $\mu$.

We denote by $M_{0}$ the family of all positive Radon measures in $X$ with compact support and by $E_{0}=E_{0}(G)$ the subfamily of $M_{0}$ constituted by positive Radon measures with finite $G$-energy. We have evidently $E_{0}(G)=E_{0}(\check{G})$.

For a compact set $K$ in $X$, we set

$$
\operatorname{cap}_{G}(K)=\sup \left\{\mu(K) ; \mu \in E_{0}, S \mu \subset K, \int G \mu d \mu \leqq \int d \mu\right\},
$$

where $S \mu$ denotes the support of $\mu$. For a subset $A$ of $X$, we denote by $\operatorname{cap}_{G}(A)$ the quantity $\sup \left\{\operatorname{cap}_{G}(K) ; K\right.$ : compact $\left.\subset A\right\}$, and we call it the $G$-capacity of $A^{1)}$. Evidently $\operatorname{cap}_{G}(A)=\operatorname{cap}_{\breve{G}}(A)$. For a subset $A$ of $X, \operatorname{cap}_{G}(A)=0$ if and only if $\left\{\mu \in E_{0} ; S \mu \subset A\right\}=\{0\}$. We say that a property holds G-p.p.p. on a subset $A$ of $X$ if $\operatorname{cap}_{G}(B)=0$, where $B$ is the set of points in $A$ where the property fails to hold.

Let us define the potential theoretical principles for a function-kernel $G$ on $X$.

\footnotetext{
1) This is usually called the inner G-capacity of $A$.
} 
(I) $G$ satisfies the restrained domination principle if, for $\mu, \nu$ in $E_{0}$, $G \mu(x) \leqq G \nu(x)$ G-p.p.p. in $X$ whenever $G \mu(x) \leqq G \nu(x)$ G-p.p.p. on $S \mu$.

Proposition 1 ([2]). Let $G$ be a strictly positive function-kernel on $X$. Then $G$ satisfies the restrained domination principle if and only if $\breve{G}$ satisfies it.

(II) $G$ satisfies the domination principle if, for $\mu$ in $E_{0}$ and $\nu$ in $M_{0}$, an inequality $G \mu(x) \leqq G \nu(x)$ on $S \mu$ implies the same inequality in the whole space.

Proposition 2. If $G$ satisfies the domination principle, then $G$ satisfies the restrained domination principle.

In fact, if for $\mu, \nu$ in $E_{0}, G \mu(x) \leqq G \nu(x) G$-p.p.p. on $S \mu$, then there exists an increasing sequence $\left(K_{n}\right)_{n=1}^{\infty}$ of compact sets contained in $S \mu$ such that $\mu\left(C K_{n}\right) \downarrow 0$ with $n \uparrow+\infty$ and $G \mu(x) \leqq G \nu(x)$ on $K_{n}$. Then, by the domination principle for $G, G \mu_{n}(x) \leqq G \nu(x)$ everywhere in $X$, where $\mu_{n}$ is the restriction of $\mu$ to $K_{n}$. Letting $n \uparrow+\infty$, we have $G \mu(x) \leqq G \nu(x)$ in $X$.

(III) $G$ satisfies the balayage principle if, for a given compact set $K$ in $X$ and a given $\mu$ in $M_{0}$, there exists a positive Radon measure $\mu^{\prime}$ in $M_{0}$ supported by $K$ such that

$$
G \mu^{\prime}(x) \leqq G \mu(x) \quad \text { on } X \text { and } \quad G \mu^{\prime}(x)=G \mu(x) \quad \text { G-p.p.p. on } K \text {. }
$$

This measure $\mu^{\prime}$ is called a $G$-balayaged measure of $\mu$ on $K$.

Proposition 3. If G satisfies the balayage principle, then $\breve{G}$ satisfies the domination principle.

This can be proved in the same way as in [3].

(IV) $G$ satisfies the complete maximum principle if for $\mu$ in $E_{0}$ and $\nu$ in $M_{0}$, an inequality $G \mu(x) \leqq G \nu(x)+1$ on $S \mu$ implies the same inequality in the whole space.

It is evident that the complete maximum principle for $G$ implies the domination principle for $G$.

(V) $G$ satisfies the classical maximum principle if for $\mu$ in $M_{0}$, an inequality $G \mu(x) \leqq 1$ on $S \mu$ implies the same inequality on $X$.

(IV) $G$ satisfies the continuity principle if for a $\mu$ in $M_{0}$, the finite continuity of the restriction of $G \mu$ to $S \mu$ implies that $G \mu$ is finite continuous in the whole space. 
When we discuss the continuity principle, it is natural to assume that a function-kernel is lower semi-continuous or continuous in the extended sense. A function-kernel $G$ on $X$ is said to be lower semicontinuous if $G$ is a lower semi-continuous function in $X \times X . G$ is called a continuous function-kernel on $X$ if $G$ is continuous in the extended sense in $X \times X$ and $G(x, y)<+\infty$ for any $(x, y)$ in $X \times X-\Delta$. The following proposition is well-known and can be proved by the same way as in the classical case (i.e., the continuity principle for the Newton kernel).

Proposition 4. If a continuous function-kernel $G$ on $X$ satisfies the domination principle or the classical maximum principle, then $G$ satisfies the continuity principle.

\section{The positivity of a continuous function-kernel and the continuity principle}

Throughout this section, $G$ is a continuous function-kernel on $X$. We say that $G$ satisfies the condition (*) if:

(*) For any non-empty open set $\omega$ in $X$, cap $\cos _{G}(\omega)>0$.

Remark. The condition (*) is very natural in the potential theory. Let us observe it for a continuous composition kernel on a locally compact abelian group.

Let $G(x, y)=k(x-y)$ be a continuous composition kernel on a locally compact abelian group $X$, where $k$ is continuous in the extended sense and finite outside the origin. Suppose that $G$ satisfies the domination principle. Then $G$ satisfies the condition $(*)$ if and only if $k$ is $\xi$-summable in a certain neighborhood of 0 , where $\xi$ is a Haar measure on $X$.

If $k$ is $\xi$-summable in a certain neighborhood of 0 , then for any finite continuous function $f$ in $X$ with compact support, the convolution $k * f$ is defined in $X$ and finite continuous. Therefore we obtain that the "if" part is valid. We shall show that the "only if" part is valid. By the domination principle for $G, k$ is identically equal to 0 if $k(0)=0$, and hence we may assume $k(0)>0$. The condition (*) implies $E_{0} \neq\{0\}$. We can find a $\lambda(\neq 0)$ in $E_{0}$ supported in $C\{0\} \cap\{x \in X ; k(x)>0\}$. We may assume that $G \lambda$ is bounded on $S \lambda$. By virtue of the domination principle for $G$, there exists a constant $c>0$ such that $G \lambda(x) \leqq c k(x)$ on 
$X$, and hence $G \lambda$ is locally bounded on $X$. Therefore, for any finite continuous function $f$ in $X$ with compact support,

$$
+\infty>\int G \lambda(x)|f(x)| d \xi(x)=\int k(x)(\breve{\lambda} *|f|)(x) d \xi(x),
$$

where $\grave{\lambda}$ is the measure defined by $\grave{\lambda}(e)=\lambda(-e)$ for any Borel set $e$. Consequently $k$ is locally $\xi$-summable.

Let us consider our continuous function-kernel on $X$. Our first theorem is the following

THEOREM 1. Let $G$ be a continuous funtion-kernel on $X$ satisfying the domination principle and the condition (*). Assume that $G>0$ on $\Delta$ and every point in $X$ is not isolated. Then $G(x, y)>0$ on $X \times X$.

Proof. Suppose that there exists a point $\left(x_{0}, y_{0}\right)$ in $X \times X$ where $G$ vanishes. Then $\left(x_{0}, y_{0}\right) \in X \times X-\Delta$. Put $g(y)=G\left(x_{0}, y\right)$. Then $g$ is defined in $X$ and continuous in the extended sense. Every point in $X$ being not-isolated, we can find $y_{1}$ in the support of $g$ satisfying $y_{1} \neq x_{0}$ and $g\left(y_{1}\right)=0$. By $G\left(y_{1}, y_{1}\right)>0$, there exists an open neighborhood $V$ of $y_{1}$ such that $G(x, y)>0$ in $V \times V$. Set $\omega=V \cap\{y \in X ; g(y)>0\}$. Then $\omega \neq \emptyset$, and there exists a positive Radon measure $\lambda(\neq 0)$ contained in $E_{0}$ and supported in $\omega$. By virtue of the Lusin theorem and the continuity principle for $G$ (cf. Proposition 4), we may assume that $G \lambda$ is finite continuous in $X$. Then we can find a positive constant $a$ such that $G \lambda(x) \leqq a G \varepsilon_{y_{1}}(x)$ on $S \lambda$, where $\varepsilon_{y_{1}}$ is the unit measure at $y_{1}$, because $G \varepsilon_{y_{1}}(x)>0$ on $S \lambda$. By the domination principle for $G$, we have $G \lambda(x)$ $\leqq a G \varepsilon_{y_{1}}(x)$ on $X$. Hence

$$
0=a G\left(x_{0}, y_{1}\right)=a G \varepsilon_{y_{1}}\left(x_{0}\right) \geqq G \lambda\left(x_{0}\right)=\int g(y) d \lambda(y)>0 .
$$

This is a contradiction. Consequently $G(x, y)>0$ in $X \times X$. This completes the proof.

We discuss the continuity principle for a continuous function-kernel on $X$. For a closed subset $X^{\prime}$ of $X$, we denote by $G^{\prime}$ the restriction of $G$ to $X^{\prime} \times X^{\prime}$. Then $G^{\prime}$ is evidently a continuous function-kernel on $X^{\prime}$.

LEMMA 1. If $G$ satisfies the domination principle, then $G^{\prime}$ satisfies it.

This follows from the fact that for any positive Radon measure $\mu$ in $X^{\prime}, G \mu(x)=G^{\prime} \mu(x)$ on $X^{\prime}$. 
Lemma 2. Suppose that $G$ is strictly positive in $X \times X$. If $G$ satisfies the restrained domination principle and the condition (*), then $G$ satisfies the continuity principle.

This lemma can be shown by the same manner as in the usual case. Let us give the proof. Suppose that for a $\mu$ in $M_{0}$, the restriction of $G \mu$ to $S \mu$ is finite continuous. It sufficies to show $\lim _{x \rightarrow x_{0}} G \mu(x)=G \mu\left(x_{0}\right)$ for every boundary point $x_{0}$ of $S \mu$.

If $\mu\left(\left\{x_{0}\right\}\right)>0, G\left(x_{0}, x_{0}\right)<+\infty$, because $G \mu\left(x_{0}\right)<+\infty$, and hence our desired equality follows immediately from the finite continuity of $G$ at $\left(x_{0}, x_{0}\right)$.

Suppose $\mu\left(\left\{x_{0}\right\}\right)=0$. By $G \mu\left(x_{0}\right)<+\infty$, for a given positive number $\varepsilon$, there exists an open neighborhood $V$ of $x_{0}$ such that $\int_{V} G\left(x_{0}, y\right) d \mu(y)$ $<\varepsilon$. The function $\int_{V} G(x, y) d \mu(y)$ of $x$ being finite continuous as a function on $S \mu \cap V$, we can choose another open neighborhood $W$ of $x_{0}$ which satisfies $W \subset V$ and

$$
\int_{W} G(x, y) d \mu(y) \leqq \int_{V} G(x, y) d \mu(y)<\int_{V} G\left(x_{0}, y\right) d \mu(y)+\varepsilon
$$

for any $x$ in $S \mu \cap W$. Denote by $\mu^{\prime}$ the restriction of $\mu$ to $W$. Then $G \mu^{\prime}<2 \varepsilon$ on $S \mu^{\prime}$. We may assume $\bar{V} \neq X$. By the condition (*) and $G(x, y)>0$ in $X \times X$, there exists a $\nu$ in $E_{0}$ such that $S \nu \cap \bar{V}=\emptyset$ and $G \nu(x)>1$ on $S \mu^{\prime}$. By virtue of the restrained domination principle for $G$ and the continuity of $G \mu^{\prime}$ in $C S \mu^{\prime}$, we have

$$
G \mu^{\prime}(x) \leqq 2 \varepsilon G \nu(x) \quad \text { in } W .
$$

On the other hand, $G\left(\mu-\mu^{\prime}\right)$ is finite and continuous at $x_{0}$, and hence

$$
\varlimsup_{x \rightarrow x_{0}} G \mu(x) \leqq G \mu\left(x_{0}\right)+2 \varepsilon G \nu\left(x_{0}\right) .
$$

$G \mu$ being lower semi-continuous and $\varepsilon$ being arbitrary, we have $\lim _{x \rightarrow x_{0}} G \mu(x)=G \mu\left(x_{0}\right)$. This completes the proof.

By Proposition 1, we have the following

LEMMA 3. Under the same assumptions as in Lemma 2, Ğ satisfies the continuity principle.

THEOREM 2. Let $G$ be a continuous function-kernel on $X$ satisfying 
the condition (*). If $G>0$ on $\Delta$ and $G$ satisfies the domination principle, then $G$ and $\breve{G}$ satisfy the continuity principle.

Proof. We denote by $X^{\prime}$ the closed subset of $X$ constituted by all points which are not isolated and by $G^{\prime}$ the restriction of $G$ to $X^{\prime} \times X^{\prime}$. By Lemma $1, G^{\prime}$ is a continuous function-kernel on $X^{\prime}$ satisfying the domination principle. Evidently $G^{\prime}$ satisfies the condition $(*)$ and every point of $X^{\prime}$ is not isolated. Consequently $\check{G}^{\prime}$ satisfies the continuity principle by Proposition 2 and Lemma 3. The continuity principle for $G$ is well-known, and we shall prove only the continuity principle for $\breve{G}$. Suppose that for $\mu \in M_{0}$, the restriction of $G \mu$ to $S \mu$ is finite continuous. Denote by $\mu^{\prime}$ the restriction of $\mu$ to $X^{\prime}$. Then, as a function on $S \mu^{\prime}$, $G^{\prime} \mu^{\prime}$ is finite continuous, because $G^{\prime} \mu^{\prime}=G \mu-G\left(\mu-\mu^{\prime}\right)$ on $X^{\prime}$, and hence $G^{\prime} \mu^{\prime}$ is finite continuous in $X^{\prime}$. By $G \mu^{\prime}=G^{\prime} \mu^{\prime}$ on $X^{\prime}$ and the fact that $X-X^{\prime}$ is discrete, $G \mu^{\prime}$ is finite continuous on $X$. On the other hand, $S\left(\mu-\mu^{\prime}\right)$ is discrete, and then $G\left(\mu-\mu^{\prime}\right)$ is finite continuous on $X$, i.e., $G \mu$ is finite continuous on $X$. This completes the proof.

\section{Remarks on Kishi's theorem}

Let us start the following theorem.

THEOREM 3. Let $G$ be a continuous function-kernel on $X$. Assume $G(x, x)>0$ on 4 . Then the following four statements are equivalent.

(1) G satisfies the domination principle.

(2) Ğ satisfies the domination priciple.

(3) $G$ satisfies the balayage principle.

(4) G satisfies the balayage principle.

Proof. By Proposition 3, we know (3) $\Rightarrow$ (2) and (4) $\Rightarrow$ (1). The implication (2) $\Rightarrow(4)$ is the dual form of (1) $\Rightarrow(3)$. Therefore it suffices to show the implication (1) $\Rightarrow(3)$. Suppose that (1) is valid. Put $\Omega=$ $\cup\left\{\omega\right.$ : open, $\left.\operatorname{cap}_{G}(\omega)=0\right\}$ and $X^{\prime}=X-\Omega$. Let $G^{\prime}$ the restriction of $G$ to $X^{\prime} \times X^{\prime}$. Then $G^{\prime}$ is a continuous function-kernel on $X^{\prime}$ satisfying the domination principle and the condition (*). Therefore, by Theorem 2 , $G^{\prime}$ satisfies the continuity principle. Let us remember the existence theorem of Kishi ([4]).

Proposition 5. Let $\bar{G}$ be a lower semi-continuous function-kernel on a locally compact Hausdorff space $Y$. Assume that $\bar{G}(x, x)>0$ for any 


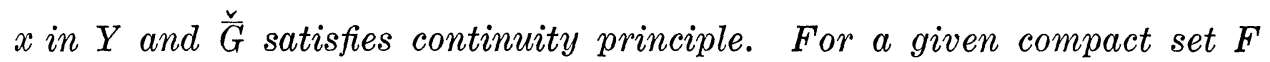
in $Y$ and a given non-negative finite continuous function $u$ on $F$, there exists a positive Radon measure $\lambda$ in $Y$ supported by $F$ such that $\bar{G} \lambda(x)$ $\geqq u(x) G$-p.p.p. on $F$ and $\bar{G} \lambda(x) \leqq u(x) \lambda$-a.e. on $Y$.

We continue our proof. Let $K$ be a compact set in $X$ and $\mu$ be a positive Radon measure in $X$ with compact support. First we suppose $S \mu \cap K=\emptyset$. Let $\mu^{\prime}$ be a positive Radon measure in $X$ obtained in Proposition 5 for the case of $\bar{G}=G^{\prime}, F=K \cap X^{\prime}$ and $u=G \mu$. We have evidently $G \mu^{\prime}(x)=G^{\prime} \mu^{\prime}(x)$ on $X^{\prime}$ and $\mu^{\prime} \in E_{0}(G)$, and " $G^{\prime}-p . p$.p. on $K \cap X^{\prime \prime}$ " is equal to "G-p.p.p. on $K \cap X^{\prime \prime}$. By virtue of the domination principle for $G$ and the inequality $G \mu^{\prime}(x) \leqq G \mu(x) \mu^{\prime}$-a.e. in $X$, we obtain, by the usual way, the inequality $G \mu^{\prime}(x) \leqq G \mu(x)$ everywhere in $X$. Consequently $\mu^{\prime}$ is a $G$-balayaged measure of $\mu$ on $K \cap X^{\prime}$. For an arbitrary $\nu$ in $E_{0}(G)$ supported by $K, \nu$ is supported by $K \cap X^{\prime}$. In fact, if $\nu(\Omega)>0$, there exists an open set $\omega \subset \Omega$ such that $\operatorname{cap}_{G}(\omega)=0$ and $\nu(\omega)>0$, which is a contradiction. Hence "G-p.p.p. on $K$ " is equivalent to " $G$ p.p.p. on $K \cap X^{\prime \prime}$. Therefore $\mu^{\prime}$ is a $G$-balayaged measure of $\mu$ on $K$.

We remark here that, to show immediately the existence of a $G$ balayaged measure of $\mu$ on $K$ in the case of $S \mu \cap K \neq \emptyset$, it is necessary that $\check{G}$ satisfies the continuity principle (cf. [3]). But, by the above discussion, we obtain that $\breve{G}$ satisfies the domination principle. In fact, suppose that for a $\nu$ in $E_{0}(G)$ and a $\lambda$ in $M_{0}, \breve{G} \nu(x) \leqq \breve{G} \lambda(x)$ on $S \nu$. For an arbitrary $y$ in $C S \nu$, there exists a $G$-balayaged measure $\varepsilon_{y}^{\prime}$ of $\varepsilon_{y}$ on $S \nu$, and hence we have

$$
\begin{aligned}
\check{G} \nu(y) & =\int G \varepsilon_{y}(x) d \nu(x)=\int G \varepsilon_{y}^{\prime}(x) d \nu(x)=\int \check{G}_{\nu}(x) d \varepsilon_{y}^{\prime}(x) \\
& \leqq \int \check{G} \lambda(x) d \varepsilon_{y}^{\prime}(x)=\int G \varepsilon_{y}^{\prime}(x) d \lambda(x) \leqq \int G \varepsilon_{y}(x) d \lambda(x)=\check{G} \lambda(y),
\end{aligned}
$$

i.e., $\check{G}_{\nu} \leqq \breve{G} \lambda$ on $X$. Consequently $\check{G}$ satisfies the domination principle, and hence $\breve{G}$ satisfies the continuity principle (cf. Proposition 4). In the same way as in [3], we obtain that $G$ satisfies the balayage principle. This completes the proof.

By the present theorem and Proposition 4, we have the following

COROLlaRY. Let $G$ be a continuous function-kernel on $X$ satisfying $G>0$ on $\Delta$. If $G$ satisfies the domination principle, then $G$ and $\breve{G}$ satisfy the continuity principle. 
M. Kishi discussed other potential theoretical properties for $G$ implied by the domination principle for $G$ under the condition that $\check{G}$ satisfies the continuity principle (cf. [3], [4], \& c.). By the above corollary, in these cases, we can omit the continuity principle for $\breve{G}$. In particular, the following theorem is fundamental.

THEOREM 4. Let $G$ be a continuous function-kernel on $X$ satisfying $G>0$ on $\Delta$. Then $G$ satisfies the complete maximum principle if and only if $G$ satisfies the domination principle and the classical maximum principle.

\section{REFERENCES}

[1] R. Durier: Thèse, Faculté des Sciences d'Orsay, Université de Paris, 1969.

[2] M. Itô: Sur les principes divers du maximum et le type positif, Nagoya Math. J., 44, 1971, p. 133-164.

[3] M. Kishi: Maximum principles in the potential theory, Nagoya Math. J., 23, 1963, p. 165-187.

[4] 1 An existence theorem in potential theory, Nagoya Math. J., 27, 1966, p. $133-137$.

Suzuka College of Technology

and

Nagoya University 